\title{
ANÁLISE CRÍTICA DAS CONSTRUÇÕES PROTÉTICAS, FIXAS E REMOVÍVEIS, SOBRE IMPLANTES EM PACIENTES EDENTADOS
}

\section{CRITICAL ANALYSIS OF THE FIXED AND REMOVABLE IMPLANT PROSTHESIS ON IMPLANTS IN EDENTULOUS PATIENTS}

\author{
Danilo Chaccur \\ Flávia M. Lopes* \\ Atlas E. Moleros Nakamae** \\ Maria Luiza Moreira Arantes Frigerio* \\ Pedro Tortamano Neto** \\ Dalva Cruz Laganá*
}

\begin{abstract}
RESUMO
A osseointegração é, sem sombra de dúvida, a responsável pelo notável avanço tecnológico, mudança de conceitos filosóficos e condução de planejamentos voltados à reabilitação oral, como também, pela disseminação e propagação dos conhecimentos, interferindo sobremaneira nas demais áreas da Odontologia contemporânea. Os implantes osseointegrados são cada vez mais utilizados para substituir dentes perdidos em várias situações, desde a perda de um único dente até em casos de completo edentulismo. A reabilitação de pacientes desdentados com próteses fixas ou overdentures removíveis é um processo complexo e desafiador. Existem fatores cruciais para a decisão de uma prótese fixa ou removível para se obter estética, fonética, promover conforto e função. Além disso, o resultado do tratamento está relacionado à taxa de sobrevivência dos implantes, à manutenção do osso marginal, à saúde do tecido periimplantar, à longevidade dos componentes protéticos e da prótese em si. Esse estudo buscou analisar as vantagens e desvantagens das reabilitações protéticas sobre implantes, fixas e removíveis, para pacientes totalmente desdentados.
\end{abstract}

DESCRITORES: Implantes dentários • Arcada edentada • Prótese dentária fixada por implantes • Prótese total.

\section{ABSTRACT}

Doubtless, osseointegration is the major responsible for the remarkable technological advance, changing philosophical concepts and guiding treatment plans related to oral rehabilitation. It also contributes to knowledge spread and diffusion, interfering in other areas of contemporary dentistry. Osseointegrated implants are widely used to replace lost teeth in many situations, since the loss of a single tooth to completely edentulous arch cases. Rehabilitation of edentulous patients by using fixed or removable implant-supported prostheses is a complex and challenging procedure. There are crucial factors involved at the decision of whether fixed or removable implant prosthesis should be planned to acquire optimal esthetics, phonetics, comfort, and function. In addition, the treatment result is related to implant survival, crest bone loss, soft tissue health, prosthesis's components longevity and prosthesis longevity itself. The aim of the present study is analyze the advantages and disadvantages of prosthetic rehabilitation of completely edentulous patients with fixed or removable implants. DESCRIPTORS: Dental implants • Jaw, edentulous • Prosthesis, dental, implant, supported • Denture, complete.

\footnotetext{
* Pós-graduandos do Departamento de Prótese da Faculdade de Odontologia da Universidade de São Paulo - USP. dchaccur@usp.br, fla-lopes@usp.br ** Professores Doutores do Departamento de Prótese da Faculdade de Odontologia da Universidade de São Paulo - USP. atlas@usp.br, mlmafrig@usp.br, tortaman@usp.br,

*** Professora Titular do Departamento de Prótese da Faculdade de Odontologia da Universidade de São Paulo - USP. dclagana@usp.br
} 


\section{N T RO DUÇÃO}

A Implantodontia trouxe avanços notáveis à Odontologia contemporânea, e a reabilitação oral com implantes tornou-se uma opção amplamente aceita e que mudou, substancialmente, a filosofia de tratamento, não só da especialidade protética, como também das demais áreas odontológicas. Os implantes osseointegrados são cada vez mais utilizados para substituir dentes perdidos em várias situações clinicas, desde a perda de um único dente até situações de perdas totais dos órgãos dentais (Henry ${ }^{1}$ 2000).

Comparada a outras áreas, a Implantodontia foi a que mais inovou e apresentou o maior avanço tecnológico nos últimos anos, desenvolvendo novos sistemas de implante, novas técnicas cirúrgicas, novos sistemas de precisão de adaptação protética, assim como o uso de tecnologias industriais do tipo CAD/CAM. Além de alavancar todos os fatores relacionados à tecnologia, a osseointegração influenciou, de maneira preponderante, a nova visão filosófica sobre a forma de diagnosticar e tratar os pacientes (Jivroj² 2005). Esse comportamento dos profissionais cirurgiõesdentistas, observado nas atuais formas de resolução dos casos, causa muitas interrogações quanto aos tradicionais princípios da reabilitação oral e os novos princípios que direcionam para outras vertentes, as quais conduzem, não raramente, à condenação de dentes, outrora mantidos no arco por meio de diversas terapias e que, hoje, encontram sua eliminação, sendo esses órgãos sumariamente trocados por implantes.

No entanto, os pacientes atualmente têm grandes expectativas estéticas e prover restaurações funcionais e confortáveis, não é mais suficiente para satisfazê-los. Mais do que nunca o diagnóstico e planejamento são fatores diretamente relacionados com o sucesso do tratamento e devem ser baseados em literatura científica com resultados consistentes (Jivroj ${ }^{2} 2005$ ).

É importante levar-se em consideração tanto as apreensões do cirurgião-dentista quanto as do paciente. Para o cirurgiãodentista, são parâmetros importantes: a taxa de sobrevivência do implante, a
ISSN 1983-5183

longevidade da prótese e a frequência de complicações. Por outro lado, para o paciente, fatores psicossociais e o custo/ benefício são fatores importantes, assim como estética, função, conforto, bem-estar e autoconfiança (Guckes et al. $\left.{ }^{3}, 1996\right)$.

Em pacientes desdentados parciais ou totais, o planejamento para reabilitação protética associada aos implantes está diretamente relacionado às condições bucais presentes. Para a definição do tipo de prótese, os problemas existentes são avaliados, determinando o que seria mais desejável: se uma reabilitação com prótese fixa ou removível sobre implante.

O protocolo fixo mandibular, estabelecido por Brånemark, apresenta resultado clínico altamente satisfatório ${ }^{4,5,6}$. Em casos de reabsorção óssea severa, principalmente em maxila, a possibilidade de se colocar implante é limitada, devido ao fino e delicado osso trabecular com uma cortical também fina e, às vezes, ausente (Jaffin e Bermann7, 1991). A proximidade com o seio maxilar pode dificultar a colocação de implante na região posterior, sendo, por vezes, necessárias reconstruções ósseas extensas anteriores à colocação do implante (Ulm et al. ${ }^{8}$ 1995). O planejamento, nessa situação clínica, pode ser resolvido com a colocação de 4 implantes na região anterior associados a uma overdenture (Karabuda et al. ${ }^{9}$ 2008). Essa modalidade protética proporciona uma melhora significativa na performance mastigatória, na estética e em fatores psicossociais (Blomberg e Lindquirt ${ }^{10}$ 1983, Muller et al. ${ }^{11}$ 2008).

Entretanto, não está claro qual tipo de prótese ou técnica clínica é superior com relação à longevidade do implante, à manutenção do osso marginal, à saúde do tecido periimplantar, à longevidade dos componentes protéticos e da prótese em si.

Este estudo tem o objetivo de analisar o resultado do uso das reabilitações protéticas sobre implantes, fixas e removíveis, para pacientes desdentados.

\section{REVISÃO DA LITERATURA E DISCUSSÃO}

Fatores relacionados à indicação de prótese fixa ou removível implanto-supor-
CHACCUR D

LOPES FM

NAKAMAE AEM

FRIGERIO MLMA

tortomano Neto P

LAGANÁ DC

ANÁLISE CRÍTICA

DAS CONSTRUÇÕES

PROTÉTICAS,

FIXAS E

REMOVÍVEIS,

SOBRE IMPLANTES

EM PACIENTES

EDENTADOS

$\therefore 241$

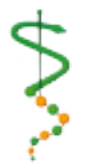

REVISTA DE ODONTOLOGIA DA UN I VERS I DADE Cidade de São PAULO

$2010 ; 22(3): 240-$

6 , SET-DEZ 
CHACCUR D

LOPES FM

NAKAMAE AEM

FRIGERIO MLMA TORTOMANO NETO P

$\angle A G A N A ́ \quad D C$

ANÁLISE CRÍTICA

DAS CONSTRUÇÕES

PROTÉTICAS,

FIXAS E

REMOVÍVEIS,

SOBRE IMPLANTES

EM PACIENTES

EDENTADOS

$242 \ldots$

REVISTA DE

ODONTOLOGIA DA

UNIVERSIDADE

CIDADE DE SÃO

PAULO

$2010 ; 22(3): 240-$

6 , SET-DEZ tadas em pacientes desdentados

É consenso que a maioria dos pacientes prefere a reabilitação com prótese fixa ou quer deixar de usar suas próteses removíveis (DeBoer ${ }^{12}$ 1993). Portanto, é importante avaliar previamente a possibilidade de corresponder à expectativa dos pacientes. Para isso, a prótese recomendada deve ser muito bem explicada e discutida com o paciente. É importante salientar que este tem o direito de ter seu espaço preservado; quer dizer: Deve-se esclarecê-lo de forma que traga o maior entendimento possível do tratamento ao qual deverá ser submetido e deixar que ele opte ou decida pelo que melhor lhe convier, evitando pressioná-lo para que não tome atitudes precipitadas ou sem que a proposta ou ideia tenha amadurecido o suficiente para a tomada de decisão e que esta não lhe traga arrependimento posterior. Em determinados casos, por exemplo, uma overdenture é a prótese mais conveniente para o paciente e erroneamente se propõe uma prótese fixa. No decorrer de tratamento muda-se o plano de tratamento para uma prótese removível. Esta conduta é, primeiramente, decepcionante para o paciente e, em segundo lugar, incômodo para o protesista que pode ter que lidar com um posicionamento inadequado dos implantes.

Portanto, fatores cruciais devem ser considerados, e serão estes que irão determinar a posição ideal dos implantes. O exame clínico extraoral dá parâmetros faciais como suporte facial, suporte labial e linha alta de sorriso. No exame intraoral deve-se avaliar: qualidade e quantidade de mucosa, quantidade e contorno ósseo, relação entre a coroa e o osso, espaço entre os arcos dentários e área fonética (Zitzmann e Marinello ${ }^{13}$ 1999).

O suporte facial é um fator crítico na decisão a ser tomada, porque o suporte do tecido mole poderá ser obtido principalmente pela parte vestibular (rósea) da prótese e pela posição dos dentes da prótese. O suporte facial torna-se mais importante ainda em pacientes com falta de volume vestibular na maxila ou para compensação de prognatismo. Ambos podem ser adquiridos devido aos padrões diferentes de reabsorção óssea da maxila e da man- díbula de pacientes desdentados.

Em pacientes dentados o suporte labial é dado pelo tecido ósseo alveolar da maxila e pela emergência cervical dos incisivos superiores. Em pacientes com maxila edêntula, o padrão de reabsorção óssea tem direção centrípeta e, portanto, a posição retruída da parte anterior da maxila frequentemente aparece (Atwood ${ }^{14}$ 1979, Desjardins ${ }^{15}$ 1992).

Para se avaliar as mudanças relativas à reabsorção óssea é necessário estimar a distância entre a linha óssea e a posição ideal das coroas clínicas.

Para a reabilitação com uma prótese fixa, a coroa clínica deve terminar no nível do tecido mole do rebordo alveolar. Nessa situação, deve ter ocorrido somente uma reabsorção mínima do tecido ósseo alveolar (quantidade de osso tipo A ou B) e deve-se ter uma ótima relação entre $\mathrm{O}$ lábio e os dentes. O paciente, nesse caso, só precisa da reposição de dentes e não de tecido mole ou ósseo (Sadowisky ${ }^{16} 1997$, Zitzmann e Marinello ${ }^{17}$ 1999). Quando, por outro lado, existe uma grande distância vertical entre a posição dos dentes artificiais e a linha do tecido mole, uma reabsorção óssea moderada ou severa da maxila deve ter ocorrido. Fazer uma prótese total fixa nessa situação pode resultar em dentes com excessivo comprimento e inclinação vestibular com espaços interproximais grandes ou pilares intermediários visíveis. Como o suporte labial não pode ser estabelecido adequadamente, a fala pode ficar prejudicada e a estética também. Mesmo com o uso de porcelana rosa para esconder a distância vertical, ela não é suficiente para dar suporte labial, que deve ser dado pela parte vestibular de uma prótese removível (Zitzmann e Marinello ${ }^{13}$ 1999).

Pacientes idosos, em particular, acham mais difícil adaptar a fala após a instalação de uma prótese fixa do que com uma nova prótese total superior (Lundquist et al. ${ }^{18}$ 1992). A falta de contato da língua com o tecido mole do palato é a primeira causa na deterioração do som do "s". A reabilitação desses pacientes com próteses removíveis permite individualizar o contorno do palato dando uma convexidade ideal entre a parte marginal da coroa 
ISSN 1983-5183

dos dentes e o tecido mole (Parel et al. ${ }^{19}$ 1986).

Recomendações diferentes são encontradas com relação ao desenho da prótese e ao número de implantes. Entretanto, evidências científicas presentes na literatura são mínimas, e a maioria apenas relata a taxa de sobrevivência dos implantes.

Nas publicações recentes, as reabilitações na mandíbula são mais predominantes do que da maxila. As próteses possuem uma infraestrutura metálica com dentes em acrílico e uma base também acrílica para compensar a perda dos tecidos mole e ósseo. Essas próteses são suportadas por um número mínimo de 4 implantes, até 6 implantes, e são parafusadas. Os implantes são colocados na região interforaminal, e para diminuir o braço de alavanca o comprimento da arcada é diminuído.

As próteses fixas da maxila requerem uma absoluta congruência entre o posicionamento do implante e do dente da prótese. Além disso, para uma prótese parafusada, é preciso que o implante seja colocado em um eixo axial adequado, ou torna-se necessário o uso de pilares intermediários angulados.

As próteses fixas para arcadas edentadas totais são complexas e uma adaptação passiva é difícil de ser obtida. Hoje a confecção de infraestruturas amplas é optimizada pela tecnologia de CAD-CAM, a qual usa titânio, e mais recentemente zircônia. Ambos os materiais têm a vantagem de menor peso em comparação às ligas de ouro. Essa tecnologia facilita a produção uniforme e precisa. Do ponto de vista técnico, parece ser desejado que a estrutura seja conectada diretamente ao implante, sem pilares intermediários. Uma adaptação passiva pode ser melhorada e a fenda entre a estrutura e o implante é minimizada (Mericske-Stern ${ }^{20}$ 2008).

\section{Prótese fixa versus overdenture}

A comparação da taxa de sucesso para próteses fixas e removíveis implanto-suportadas é crítica, já que o primeiro fator para seleção de uma overdenture está no fato de se ter um osso de pobre qualidade e com grande reabsorção, o que resulta num número limitado de implantes curtos $21,22,23,24$.
A perda de implante está relacionada com uma quantidade e qualidade óssea pobres (Engquirt et al. ${ }^{21}$ 1988, Hutton et al. ${ }^{25}$ 1995). Essa hipótese tem sido apoiada por resultados clínicos reportados pela instalação de próteses fixas sobre somente 4 implantes com comprimento de 7 a 10 mm. O risco de o implante falhar foi significantemente maior nas situações em que 4 implantes foram colocados ao invés de 6. Em mais do que $60 \%$ das próteses parafusadas sobre 4 implantes houve falha do implante num período de observação de 10 anos (Jemt e Lekholm ${ }^{26}$ 1995).

Bryant e colaboradores numa revisão sistemática, incluindo meta-análise, procuraram analisar o impacto do tipo de prótese fixa ou removível na taxa de sobrevivência dos implantes e no sucesso. Encontraram uma taxa de sobrevivência do implante $6,6 \%$ maior em próteses fixas na mandíbula do que na maxila. Observaram uma taxa de falha no implante maior nas próteses removíveis (overdentures) na maxila, aparentemente relacionada com um volume ósseo deficiente. Apesar de este estudo sugerir que a taxa de sobrevivência dos implantes e o sucesso não serem afetados pelo tipo de prótese, concluíram que a demanda de manutenção pode variar com o tipo de prótese, principalmente com relação aos encaixes das overdentures (Branemark et al. ${ }^{27}$ 1995).

Zitzmann e Marinello, em um estudo em perspectiva, compararam os resultados do tratamento com prótese implanto-suportada em maxila edêntula, fixa e removível. Encontraram uma taxa de sobrevivência do implante de 97,6\% em próteses fixas parafusadas e 94,4\% em overdentures na maxila. Nos dois grupos, o nível ósseo e a saúde periimplantar e a higiene oral não foram considerados significantes. Concluíram que a maior parte das complicações mecânicas pôde ser resolvida durante as consultas de controle e não foram necessárias mais consultas. Os autores sugeriram que os pacientes sejam chamados para controle a cada 6 meses e, dessa forma, pode-se prevenir problemas biológicos ou mecânicos mais graves que possam ocorrer (Bryant et al. ${ }^{28}$ 2007).

Em outro estudo, Zitzmann e Marinello compararam dois tipos de prótese (fixa e
CHACCUR D

LOPES FM

NAKAMAE AEM

FRIGERIO MLMA

tortomano Neto P

LAGANÁ DC

ANÁLISE CRÍTICA

DAS CONSTRUÇÕES

PROTÉTICAS,

FIXAS E

REMOVÍVEIS,

SOBRE IMPLANTES

EM PACIENTES

EDENTADOS

$\therefore 243$

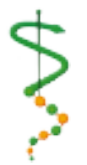

REVISTA DE

ODONTOLOGIA DA

UN I VERS I DADE

Cidade de São

PAULO

$2010 ; 22(3): 240-$

6 , SET - DEZ 
CHACCUR D

LOPES FM

NAKAMAE AEM

FRIGERIO MLMA TORTOMANO NETO P

LAGANÁ DC

ANÁLISE CRÍTICA

DAS CONSTRUÇÕES

PROTÉTICAS,

FIXAS E

REMOVÍVEIS,

SOBRE IMPLANTES

EM PACIENTES

EDENTADOS

$244 \cdots$

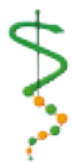

REVISTA DE

ODONTOLOGIA DA

UNIVERSIDADE

CIDADE DE SÃO

PAULO

$2010 ; 22(3): 240-$

6 , SET-DEZ
ISSN 1983-5183

removível em maxila edêntula) com ênfase no ponto de vista do paciente. Os dois tipos de prótese foram associados com uma melhora significativa quanto ao conforto e retenção, função, estética e aparência, paladar, fala e autoestima (Zitzmann e Marinello ${ }^{29}$, 2000).

Quanto a complicações no tecido mole, em próteses removíveis sobre implante na maxila, hiperplasia gengival foi observada em $14 \%$ dos pacientes ( $7 \%$ dos implantes) e foi atribuída ao comprimento insignificante do pilar intermediário selecionado (Zitzmann e Marinello ${ }^{29}$, 2000). Pilares intermediários com margem supragengival foram indicados para melhorar o acesso para a higiene oral com fio dental e escovas interproximais pequenas (Zitzmann e Marinello ${ }^{13}$ 1999).

Dependendo da extensão palatina da prótese, somente uma pequena quantidade de saliva penetra na região peri-implante. Esses "espaços mortos" ocorrem quando são usadas barras pré-fabricadas com várias distâncias do tecido mole e quando a prótese é aliviada ao redor de elementos retentivos. Sob essas circunstâncias, vários microrganismos patogênicos são encontrados em número maior em próteses removíveis do que em fixas (Chan et al. ${ }^{31}$ 1998).

Problemas mecânicos associados às próteses fixas são a fratura do parafuso de ouro, do parafuso do pilar intermediário, ou mesmo da infraestrutura, os quais presume-se como resultado de falta de adaptação passiva da infraestrutura e/ou inapropriado carregamento do implante devido à colocação imprópria do implante ou hábitos parafuncionais (DeBoer ${ }^{12}$ 1993, Smedberg32 1993).

Quando avaliados a duração e o custo associados à manutenção de próteses fixas ou removíveis, deve-se considerar que o custo para recolocar um clip ou reativar um encaixe é insignificante quando comparado à necessidade de reaplicação de material estético, reposição do parafuso de pilar intermediário, ou de uma estrutura fraturada.

\section{CONCLUSÃO}

Uma análise crítica com base na literatura atual da relação dos resultados dos tratamentos com prótese fixa ou overdenture implanto-retidas foi apresentada para melhor entender as diferenças e as desvantagens de cada método. Pelo fato de cada situação ser única, todos os parâmetros discutidos previamente devem ser cuidadosamente estudados para preencher as necessidades e expectativas dos pacientes, assim como estabelecer um resultado biológico e funcional a longo prazo.

\section{REFERÊNCIAS}

1. Henry PJ. Tooth loss and implant replacement. Aust Dent J 2000 Sep; 45(3): 150-72

2. Jivraj SA. Treatment planning in implant dentistry. An introduction to the issue. J Calif Dent Assoc 2005 Apr; 33(4): 289-90

3. Guckes AD, Scurria MS, Shugars DA. A conceptual framework for understanding outcomes of oral implant therapy. J Prosthet Dent 1996 Jun; 75(6): 633-9

4. Albrektsson T, Zarb G, Worthington P, Eriksson AR. The long-term efficacy of currently used dental implants: a review and proposed criteria of success. Int J Oral Maxillofac Implants 1986; 1(1): 11-25

5. Albrektsson T, Sennerby L. State of the art in oral implants. J Clin Periodontol 1991 Jul; 18(6): 474-81.

6. Esposito M, Hirsch JM, Lekholm U, Thomsen P. Biological factors contributing to failures of osseointegrated oral implants. (I). Success criteria and epidemiology. Eur J Oral Sci 1998 Feb; 106(1): 527-51. 
7. Jaffin RA, Berman CL. The excessive loss of Branemark fixtures in type IV bone: a 5-year analysis. J Periodontol 1991 Jan; 62(1): 2-4

8. Ulm CW, Solar P, Gsellmann B, Matejka M, Watzek G. The edentulous maxillary alveolar process in the region of the maxillary sinus--a study of physical dimension. Int J Oral Maxillofac Surg 1995 Aug; 24(4): 279-82

9. Karabuda C, Yaltirik M, Bayraktar M. A clinical comparison of prosthetic complications of implant-supported overdentures with different attachment systems. Implant Dent 2008 Mar; 17(1): 74-81

10. Blomberg S, Lindquist LW. Psychological reactions to edentulousness and treatment with jawbone-anchored bridges. Acta Psychiatr Scand 1983; 68(4): 251-62

11. Muller K, Morais J, Feine J. Nutritional and anthropometric analysis of edentulous patients wearing implant overdentures or conventional dentures. Braz Dent J 2008; 19(2): $145-50$

12. DeBoer J. Edentulous implants: overdenture versus fixed. J Prosthet Dent 1993 Apr; 69(4): 386-90

13. Zitzmann NU, Marinello CP. Treatment plan for restoring the edentulous maxilla with implant-supported restorations: removable overdenture versus fixed partial denture design. J Prosthet Dent 1999 Aug; 82(2): 188-96

14. Atwood DA. Bone loss of edentulous alveolar ridges. J Periodontol 1979; 50(4): 11 21

15. Desjardins RP. Prosthesis design for osseointegrated implants in the edentulous maxilla. Int J Oral Maxillofac Implants 1992; 7(3): 311-20

16. Jemt T, Lekholm U. Implant treatment in edentulous maxillae: a 5-year follow-up report on patients with different degrees of jaw resorption. Int J Oral Maxillofac Implants 1995 mai-jun; 10(3): 303-11

17. Zitzmann NU, Marinello CP. Clinical and technical aspects of implant-supported restorations in the edentulous maxilla: the fixed partial denture design. Int J Prosthodont 1999 Jul-Aug; 12(4): 307-12

18. Lundqvist S, Lohmander-Agerskov A, Haraldson T. Speech before and after treatment with bridges on osseointegrated implants in the edentulous upper jaw. Clin Oral Implants Res 1992 Jun; 3(2): 57-62

19. Parel SM, Balshi TJ, Sullivan DY, Cardenas ER. Gingival augmentation for osseointegrated implant prostheses. J Prosthet Dent 1986; 56(2): 208-11

20. Mericske-Stern R. Prosthetic considerations. Aust Dent J 2008; 53 Suppl 1:S49-59

21. Engquist B, Bergendal T, Kallus T, Linden U. A retrospective multicenter evaluation of osseointegrated implants supporting overdentures. Int J Oral Maxillofac Implants 1988; 3(2): 129-34

22. Jemt T, et al. A 5-year prospective multicenter follow-up report on overdentures supported by osseointegrated implants. Int J Oral Maxillofac Implants 1996 May-Jun; 11(3): 291-8

23. Naert I, Quirynen M, Theuniers G, van Steenberghe D. Prosthetic aspects of osseointegrated fixtures supporting overdentures. A 4-year report. J Prosthet Dent 1991 May; 65(5): 671-80

24. Palmqvist S, Sondell K, Swartz B. Implant-supported maxillary overdentures: outcome in planned and emergency cases. Int J Oral Maxillofac Implants 1994 Mar-Apr 9(2): 184-90
CHACCUR D

LOPES FM

NAKAMAE AEM

FRIGERIO MLMA

TORTOMANO NETO P

LAGANÁ DC

ANÁLISE CRÍTICA

DAS CONSTRUÇÕES

PROTÉTICAS,

FIXAS E

REMOVÍVEIS,

SOBRE IMPLANTES

EM PACIENTES

EDENTADOS

245

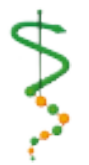

REVISTA DE

ODONTOLOGIA DA

UNI VERS I DADE

CIDAdE DE SÃo

PAULO

$2010 ; 22(3): 240-$

6 , SET-DEZ 
CHACCUR D

LOPES FM

NAKAMAE AEM

FRIGERIO MLMA

TORTOMANO NETO $P$

$\angle A G A N A ́ \quad D C$

ANÁLISE CRÍTICA

DAS CONSTRUÇÕES

PROTÉTICAS,

FIXAS E

REMOVÍVEIS,

SOBRE IMPLANTES

EM PACIENTES

EDENTADOS

\section{6}

I SSN 1983-5183

25. Hutton JE, et al. Factors related to success and failure rates at 3-year follow-up in a multicenter study of overdentures supported by Branemark implants. Int J Oral Maxillofac Implants 1995 Jan-Feb; 10(1): 33-42

26. Sadowsky SJ. The implant-supported prosthesis for the edentulous arch: design considerations. J Prosthet Dent 1997 Jul; 78(1): 28-33

27. Branemark PI, Svensson B, van Steenberghe D. Ten-year survival rates of fixed prostheses on four or six implants ad modum Branemark in full edentulism. Clin Oral Implants Res 1995 Dec; 6(4): 227-31

28. Bryant SR, MacDonald-Jankowski D, Kim K. Does the type of implant prosthesis affect outcomes for the completely edentulous arch? Int J Oral Maxillofac Implants 2007; 22 Suppl: 117-39

29. Zitzmann NU, Marinello CP. Treatment outcomes of fixed or removable implantsupported prostheses in the edentulous maxilla. Part II: clinical findings. J Prosthet Dent 2000 Apr; 83(4): 434-42

30. Zitzmann NU, Marinello CP. Treatment outcomes of fixed or removable implant-supported prostheses in the edentulous maxilla. Part I: patients' assessments. J Prosthet Dent 2000 Apr; 83(4): 424-33

31. Chan MF, Narhi TO, de Baat C, Kalk W. Treatment of the atrophic edentulous maxilla with implant-supported overdentures: a review of the literature. Int J Prosthodont 1998; 11(1): 7-15

32. Smedberg J-I, Svensäter G, Edwardsson S. The microflora adjacent to osseointegrated implants supporting maxillary removable prostheses. Clin Oral Implants Res 1993 4(4): 165-71

Recebido em: 19/09/2010

Aceito em: 16/03/2010 